\title{
TREATMENT IN ACUTE LARGE BOWEL OBSTRUCTION DUE TO CARCINOMA
}

\author{
By Edward G. MuIR, F.R.C.S. \\ Surgeon to H.M. Household and King's College Hospital
}

In its many aspects this is a common subject in surgical literature and with good reason. Malignant disease of the colon and rectum accounts annually for some 16,000 deaths in England and Wales and the operative mortality of these cases varies considerably whether surgery is elective and planned or undertaken as an emergency measure.

\section{Site of Obstruction}

All accounts show that acute obstruction is more common with growths in the left colon and that the sigmoid is much the most frequent site. In 75 cases of acute obstruction due to malignant disease of the large bowel recently reviewed 33 or 44 per cent. of the whole, were caused by growths in the sigmoid colon. Among I 44 cases of sigmoid carcinoma 28.9 per cent. were not admitted to hospital until they were suffering from acute obstruction while similar figures for growths in other sites were Right Colon, 8.2 per cent., Left Colon, 2 r.6 per cent., and Rectum, 3.5 per cent.

\section{Treatment}

The lethal factors in large bowel obstruction appear to be the rise in pressure inside the large bowel above the growth with its effects on the bowel wall and the biochemical disturbance which occurs when the effects of the obstruction are transmitted to the small bowel. The first principle in treatment must be the decompression of the bowel and the removal of the growth must be a secondary consideration. It is occasionally possible to combine these objects but such a compromise can be dangerous. All cases require the correction of dehydration, seldom marked when first seen, the maintenance of fluid balance and gastric suction.

\section{Plain X-ray of the Abdomen}

This is now a routine first step in the treatment of any case of suspected obstruction. It will confirm that obstruction exists, that it affects the large bowel and it will indicate whether there is a competent ileo-caecal walve with a grossly distended caecum or whether back pressure is transmitted to the small bowel. In many cases it will indicate the actual site of the obstruction.

\section{Early or Subacute Obstruction}

When obstruction is early, intermittent or partial, the patient's condition satisfactory and an enema produces some flatus, it is legitimate to hope that repeated colonic washouts may decompress the bowel sufficiently to turn a case of emergency surgery into a planned operation. This is not a frequent occurrence. However, if the case shows some improvement the surgeon may feel that a barium enema becomes a justifiable investigation. It must be carefully administered and carefully washed out. It is open to the criticism that it may precipitate complete obstruction but the information obtained may be most useful. Delay in operating upon a patient with large bowel obstruction can only be justified if there is definite evidence of continued improvement.

\section{The Miller-Abbott Tube}

The successful passage of a Miller-Abbott tube can be a life-saving measure in paralytic ileus but it does not occupy a similar place in the treatment of large bowel obstruction. Much time may be lost in an attempt to negotiate the tube past the pylorus, and that in spite of all the tricks with mercury in the bag and positioning the patient and an attendent radiographer. Meanwhile the pressure in the caecum may be building up to a disastrous level. It is used by some as a useful preliminary to elective surgery in unobstructed cases though the little more it has to add there does not seem to justify it over the more convenient gastric tube. The only valid objection to its use in large bowel obstruction is that it may cause delay and once the necessary preliminaries of $\mathrm{X}$-ray, gastric suction and intravenous fluids have been completed some form of operative decompression should be performed. 


\section{'Blind' Decompression or Exploration}

Some surgeons contend that every case of intestinal obstruction will stand a quick laparotomy; others that in acute obstruction this is never justified. It is reasonable to base the decision on the patient... A laparotomy has obvious advantages; it reveals the site of the growth and gives a guide to its future operability; it confirms the diagnosis and reveals the odd or unusual case where perhaps small bowel obstruction is also present and it permits the surgeon to site his bowel drainage exactly where he wishes it to be relative to the growth. A 'blind' decompression does none of these things but it avoids the manipulation of distended bowel and perhaps the struggle to close a difficult abdomen. It always means a second operation and when in due course this shows an inoperable growth one cannot help regretting that a laparotomy and perhaps a permanent colostomy were not performed on the first occasion. As against this it may not be easy to assess operability in obstruction, and some fixity may disappear after proximal drainage. It the patient is fit and not grossly distended a laparotomy is desirable: if the condition is poor and distension marked then decompression alone should be performed.

\section{Caecostomy}

This method of bowel drainage has both advantages and disadvantages and those who dislike it find little in its favour. A distended caecum can be difficult to handle and attempts to deliver it through the abdominal wall in a stout subject may tear the wall; tube drainage of the caecum in the gastrostomy fashion may permit leakage and peritoneal soiling; it relieves tension in the caecum but it does not stop the faecal stream from passing on into the large bowel and when the second operation is performed the bowel above the growth, particularly if the left colon, may be heavily loaded. Difficulty with a distended caecum can be greatly lessened by using a needle and sucker on the bowel before any attempt is made to deliver it, for a great deal of the distension is due to gas and drainage of an 'exteriorized' caecum lessens the risk of peritoneal soiling. Repeated caecostomy washouts will help to empty the bowel. The advantage of a caecostomy is that it can be performed 'blind', if necessary under a local anaesthetic, on a patient perhaps unsuitable for anything else. If a laparotomy has been performed, a caecostomy may be chosen for obstructing growths of the ascending or transverse colon. For those in the remainder of the large bowel, a right transverse colostomy has more advantages. Some published figures give caecostomy a high mortality. That has not been my personal experience where the mortality of caecostomy at II.I per cent has been almost the same as transverse colostomy (12.5 per cent.) but the former has tended to include the worst cases. A caecostomy does not interfere with the subsequent resection of the right colon for it is easity removed with the bowel and if it has been used for the left colon it is a safety vent after the resection.

\section{Colostomy}

This not only relieves the tension in the obstrueted bowel, but it diverts the faecal stream and defunctions the bowel beyond. By colostorify washouts it is usually possible to make the future operation site clean and empty. The ideal sitenis the right part of the transverse colon, immediately beyond the hepatic flexure and here it is the best decompressive operation for any operable growth in the large bowel other than those in the right colon and right half of the transverse colon. small transverse incision is convenient, at least one inch below the costal margin and lateral to the rectus sheath. The attachment of the omentumis dissected off the colon for two or three inches and a simple loop colostomy performed with the omentum tucked around it under the anterior abdominal wall. As an alternative the incision may be mafle through the rectus sheath or the colostomy mage in the ascending colon, but the first is more diffact to close and the second more difficult to perfogrm.

The advantage of selecting a right transogerse colostomy as a decompressive measure for le lett colon growths is that it is well away fromôthe future operation site and leaves plenty of bowel length available for the future resection ofoiny growth. The resection will be performed on rogfunctioning bowel and the risks will be greaty reduced.

Just as there are advantages in placing a colō tomy as far from the growth as possible, so thepe are also some advantages in placing it in proximit, provided, that it is not near enough to disturb the growth. A ' near' colostomy means that there is $\overline{\text { mo }}$ risk of a loaded bowel at the subsequent resection and when this is performed the colostomy is resected with the growth. It obviates the thi operation to close the colostomy though the anastomosis no longer has the safety of a colostority above it. A 'near' colostomy can only be contemplated after laparotomy has shown the exact site of the growth and its operability and it has the disadvantage that it takes up bowel 'slack' in the region of the future resection. At the present tine the majority of surgeons favour a colostomy as far as possible from the future operation site. A colostomy in the left part of the transverse coles interferes with the mobilization of the splente flexure and prolapse of the proximal loop can occur in this site. A left iliac colostomy is usualty 
reserved for an inoperable growth of the sigmoid colon or rectum.

\section{Entero-anastomosis}

Attempts to relieve obstruction by shortcircuiting a growth of the colon carry a much higher mortality than simple decompressive measures. A short-circuit in the presence of obstruction is the operation of choice when laparotomy has shown an inoperable growth of the right colon, an alternative to caecostomy for an operable growth of the right colon and it is probably unwise to employ it elsewhere. An alternative in obstruction for an operable growth in the right colon is to divide the ileum nine inches proximal to the ileocaecal valve, implant the proximal end into the transverse colon and bring the distal end out through a stab drain in the right iliac fossa; a rubber tube or catheter being passed down the ileum into the caecum.

\section{Resection}

It is an old rule, unaltered by sulphonamides and anti-biotics, that primary resection and anastomosis should not be performed in the presence of obstruction. The patient's general condition does not favour a prolonged operation and the local condition of the obstructed bowel does not make for a secure anastomosis. It was for these reasons and the desire to avoid a second major operation, that resection by the Paul-Mikulicz operation was frequently used in obstruction. The main criticism of this operative method is that the necessity to bring out the two ends of bowel through the abdominal wall may lead the surgeon to a less radical resection both of the lymphatic field and of the bowel. It is not the ideal planned operation for bowel cancer but it remains a most useful proceeding when local or general conditions call for something less. Its mortality will almost certainly be higher than simple decompression but against this can be set the avoidance of a second major operation.

In certain cases of obstruction due to an operable growth of the right colon there is something to be said for occasionally breaking the rules and performing a resection with anastomosis but with the safeguard of ileal drainage. Right hemicolectomy involves not the anastomosis of large bowel above an obstruction with large bowel below but the anastomosis of small bowel to undistended large bowel. If this is combined with temporary drain- age of the small bowel it becomes a much safer procedure. After the resection of the right colon the end of the ileum is closed and a side-to-side anastomosis performed or the end of the ileum is implanted into the colon. A long rubber tube is now passed up the divided end of colon, through the anastomosis and up the terminal ileum for several inches. The divided colon is turned in around the tube like a gastrostomy and the tube brought out through a stab drain in the right iliac fossa. The colon is sutured to parietal peritoneum around the stab incision and with post-operative suction to both the ileostomy and the gastric tubes distension can be greatly reduced. This operation is a useful proceeding when an operable right colon growth is associated with early obstruction or when right colectomy is found to be unexpectedly required. If there is doubt as to the patient's fitness decompression alone should be employed.

\section{Summary}

If the patient's condition is poor a 'blind' decompression should be performed: if fit a laparotomy is to be perferred. If operable and in the right colon there is a choice of caecostomy, a short-circuit or in early fit cases a right hemicolectomy with ileostomy drain. In the transverse colon a caecostomy is better than a colostomy since mobilization of the hepatic flexure may later be required. For the splenic flexure and beyond there is a choice of caecostomy or right transverse colostomy with the latter preferred. If the growth is inoperable a short-circuit may be performed for the right colon and right half of the transverse colon and elsewhere a permanent colostomy a short distance above the growth.

\section{Results}

Thirty years ago the operative mortality of this type of acute obstruction was quoted in one large series as 43.5 per cent. In recent papers it has varied between 15.2 per cent. and 42 per cent. In fact the mortality is still alarmingly high in spite of modern advances. We are dealing with an elderly age group, a serious disease and a dangerous complication, and it is small wonder that the mortality is high. The operative mortality for unobstructed bowel carcinoma is probably in the region of 10.0 per cent. in general hands. It is safe to say that this mortality is doubled once obstruction has appeared.

\section{This issue of The Postgraduate Medical Journal completes Volume 32 Why not have your copies bound up into a permanent record?-See page 624 for details}

